Mid-American Review of Sociology

1975 "Twelve Propositions on Productivity and War Economy." Armed Forces and Society. 1:490-497.

Perlo, V.

1963 Militarism and Industry. New York: International Publishers.

Russet, B.

1967 "Who Pays For Defense." American Political Science Review. 63:412-426.

Spanier, J. and E. Ulsamer

1974 How American Foreign Policy is Made. New York: Praeger.

Stevenson, P.

1973 "The Military-Industrial Complex: An Examination of the Nature of Corporate Capitalism in America." Journal of Political and Military Sociology. 1:247-259.

Yarmolinsky, A.

1972 "The President, the Congress, and Arms Control." In S. Sarkesian (ed.), The Military-Industrial Complex: A Reassessment. Beverly Hills, Calif.: Sage.

\section{NOTES AND COMMENTS}

Dear Editor:

Please consider the enclosed paper, "On the Relationship between Dummy Variable Regression and Multiple Classification Analysis," for publication in your journal.

A note on its genesis is in order. The paper was written for the benefit of my colleagues at the Center for the Study of American Pluralism who on occasion have used these two methods in the past. The paper was very well received and one of our staff gave me a notice concerning your publication, suggesting that the paper might be of general interest to graduate students in the social sciences. Since the paper was written emphasizing the functional relationship between the two methods, I agreed that this was indeed the best audience for the discussion and example presented.

One of the perennial problems encountered in data analysis, and sample survey data analysis in particular, is estimating predictive models which employ a metric predicted variable and categorical (nonmetric) predictors. The method designed to handle this problem most often discussed in elementary statistics texts is analysis of variance (ANOVA). ANOVA however is actually a generic term for a number of estimation strategies employing categorical predictors. The type of ANOVA discussed in a beginning statistics text is only the simplest of these and is applicable only under very restrictive conditions. The more complex forms of ANOVA are less restrictive in their assumptions, but suffer the drawback of being inferential only. That is, they test statistical significance (the F-test) and estimate the variance explained in the predicted variable, but do not estimate the pattern or size of the effects of the predictors. Since pattern and size of effect are just as important as statistical significance, ANOVA alone often falls short of a researcher's goals.

Two methods of solving this last problem of estimating the effects of categorical predictors, as well as their statistical significance, are dummy variable regression (DVR) and multiple classification analysis (MCA). The former is a special case of 
multiple regression, while the latter is an extension of a particular type of analysis of variance. Despite their different origins, they are highly interrelated. The purpose of this paper is to explore this interrelationship.

\section{ASSUMPTIONS OF DVR AND MCA}

Dummy variable regression and multiple classification analysis entail identical assumptions. Both assume a single metric predicted (dependent) variable. Predictor (independent) variables should be categorical variables or some combination of categorical and metric variables. The relationship between the metric predictors and the predicted variable is assumed to be linear. No specific function is assumed for the relationship between the categorical predictors and the predicted variable. If all of one's predictors are metric, neither method is particularly useful. One should probably employ some form of multiple regression in such a case.

DVR and MCA estimate additive models. That is, interactions among the predictors are assumed nonexistent. An interaction is said to exist when the effect of any one predictor varies in strength with the levels of the other predictors in the model. Though noninteraction is assumed, neither method requires independence among the predictors, as is the case in simple ANOVA.

In terms of causal structure, both methods estimate recursive models. It is assumed that the predictors influence the predicted variable, but that the predicted variable has no influence upon the predictors. No causal hierarchy among the predictors needs to be specified in either DVR or MCA.

\section{METHOD TERMINOLOGY}

DVR and MCA differ somewhat in their terminology due to their different origins. DVR uses regression terminology, while MCA often uses that of analysis of variance. Since ANOVA terminology is a bit more specific, and since this specificity will lead to less confusion later in the paper, ANOVA terminology shall be used.
The metric predicted (dependent) variable is called the criterion variable. Categorical predictors are termed factors while metric predictors are covariates.

\section{DEFINITION OF EFFECTS}

The definition of an effect is extremely important for both DVR and MCA, since it is in this way that they differ from ANOVA. It should be noted that since both methods also entail an estimate of the variance explained in the criterion variable and generate F-ratios (or the sums of squares terms necessary to compute F-ratios), both methods implicitly perform an ANOVA as well. The estimation of effects is over and above this implicit ANOVA.

DVR and MCA predict values on the criterion variable according to the information contained in the factors and covariates used. The effect of a covariate upon the criterion is the slope, either standardized or unstandardized, of the ordinary least squares regression line controlling for the other factors and covariates in the model. This slope will be identical to that generated by a standard multiple regression and is the same for both DVR and MCA. Of the two MCA programs known to the author, SPSS and OSIRIS, only the SPSS version allows covariates.

However, fitting slopes is meaningless when estimating the effect of a factor. All members of a single factor category are treated as equal. There will be a single predicted value on the criterion variable for each category of a factor holding constant the other factors and covariates in the model.

Both DVR and MCA estimate identical predicted values for each category of each factor in a given model. Both methods will therefore explain identical proportions of the variance in the criterion variable. Since the predicted values of the categories of a particular factor are not assumed to follow a specific function, a reference point is needed with which to measure effects. Since DVR and MCA estimate identical effects for covariates and identical predicted values for factor categories, the primary difference between the methods lies in the reference point used to measure factor effects, i.e., their definition of factor effects. 
$M C A$

MCA shall be discussed first. Its reference point is the grand mean of the criterion variable. The effect of a factor is measured in relation to how well that factor's categories predict the criterion with respect to the grand mean. In order to provide a measure of this, MCA presents a coefficient called beta (called eta when only a single factor and no covariates are used) which is always positive and, except in extreme cases, is less than "one." It is defined as the positive square root of the ratio of the sum of squares due to the factor adjusted for the other factors and covariates in the model over the total sum of squares in the criterion variable. Beta, like its namesake in multiple regression-the standardized regression coefficient-is interpreted only as a measure of relative importance. However, since no specific function has been fit between the factor and the criterion variable, the sign of an MCA beta is meaningless. Hence it always has a positive sign. This is not to say that the significance of a given MCA beta cannot be established. Each beta has an associated F-ratio.

In addition to the beta statistic which allows the researcher to assess the predictive strength of a factor, MCA also generates a coefficient (a) for each category of the factor. $a$ is also measured with respect to the grand mean of the criterion variable $(\mathrm{Y})$ and is defined as:

$$
a_{i j}=\widehat{Y}_{i j}-\bar{Y}
$$

where $\hat{Y}_{i j}$ is the predicted value on the criterion variable for the $\mathrm{j}^{\text {th }}$ category of the $\mathrm{i}^{\text {th }}$ factor and $\bar{Y}$ is the grand mean of the criterion variable. These a coefficients therefore allow the researcher to assess the pattern of the effect of any given factor, controlling for the remaining factors and covariates in the model.

Unfortunately, the significance of any given a coefficient cannot be established (for a dissenting opinion, see Andrews et al. 1973:50-52). An a coefficient is estimated for every category of any given factor. By using a constant reference $(\bar{Y})$ in defining the as, the value of $a$ for the last category of a particular factor can be determined from the values taken by the previously estimated as.
This is because the weighted sum of the as will always equal zero in MCA. Therefore, if there are $n$ categories in a factor, there are $n-1$ degrees of freedom in the categories of that factor. The significance of the a coefficient can therefore not be established since there will always be one more coefficient than available degrees of freedom. The a coefficients should be interpreted as illustrative only.

To summarize, MCA measures and tests the strength of association between each factor viewed as a whole and the criterion variable. The measure is called beta and the test is the F-test. In addition, the pattern of predicted values about the grand mean of the criterion variable is shown through the $a$ coefficients associated with each factor. However, these $a$ coefficients cannot be tested.

\section{$D V R$}

Whereas MCA focuses upon testing the overall strength of a factor in predicting the criterion variable, DVR is concerned with testing the pattern of factor category effects. In order to do this, one breaks each factor down into a number of "dummy variables," each dummy variable representing a single category of the original factor. Dummy variables are dichotomies usually scored "one" if a case equals the category represented by the dummy variable, and "zero" if not. The criterion variable is regressed onto these dummy variables along with any and all covariates, hence DVR's name.

The reader will recall that the MCA measure which illustrates the pattern of category effects, the $a$ coefficients, cannot be tested for significance because there is always one more a coefficient than degrees of freedom in the categories of each factor. If dummy variables were constructed for every category of each factor, a similar problem would result. Indeed, with all categories of each factor represented by dummy variables, these dummy variables would be perfectly multicolinear and the normal regression equations would therefore be unsolvable.

In order to bring the number of dummy variables for each factor into line with the number of degrees of freedom in each 
factor, one dummy variable from each factor is omitted from the regression. The category which the omitted dummy variable represents is called the "reference category." From a mathematical standpoint, the choice of a reference category is completely arbitrary.

However, the omitted dummy variable, and thus the reference category, plays an important role in defining what is meant by an effect. The resulting unstandardized regression coefficient (b) associated with each dummy variable represents the difference in predicted value between the category represented by the dummy variable and the reference category of the factor from which the dummy variable was constructed. That is,

$$
b_{i j}=\widehat{Y}_{i j}-\widehat{Y}_{i o}
$$

where $\hat{Y}_{\mathrm{ij}}$ is the predicted value on the criterion variable $(Y)$ of the $\mathrm{j}^{\text {th }}$ category of the $\mathrm{i}^{\text {th }}$ factor, and $\hat{\mathrm{Y}}_{\mathrm{io}}$ is the predicted value on $\mathrm{Y}$ of the reference category (o) of the $i^{\text {th }}$ factor. The effect of the reference category is defined as zero since the above equation becomes

$$
b_{\text {io }}=\widehat{Y}_{\text {io }}-\widehat{Y}_{\text {io }}=0
$$

when $\mathrm{j}$ equals the reference category.

Each regression coefficient $b$ has an associated F-ratio. Therefore one can test whether or not the difference in predicted value between a given category and the reference category is significant controlling for the other factors and covariates in the model. Lest one believe that all information about the reference category has been lost, the predicted value of the reference category can be obtained by using the $\mathrm{Y}$ intercept generated by the regression routine.

Unfortunately, unlike MCA, the strength of a factor taken as a whole cannot be estimated with DVR except in the case when the original factor is a dichotomy. In such a case, the factor is represented by a single dummy variable whose standardized regression coefficient (beta) will have an absolute value identical to the value of beta generated by a MCA routine.
To summarize the difference between MCA and DVR, MCA estimates and tests the overall strength of factors with the pattern of effects provided through the $a$ coefficients. These $a$ coefficients are mere artifacts of the method and cannot be tested. On the other hand, DVR estimates and tests the pattern of effects in relation to a reference category for each factor. The overall strength of a factor can be determined only when the factor is a dichotomy. Since both methods predict identical values on the criterion variable, the unstandardized regression coefficients generated by dummy variable regression can be converted into MCA $a$ coefficients and vice versa. That is, when the dummy. variables are scored zero-one,

$$
b_{i j}=a_{i j}-a_{i o}
$$

where $a_{i o}$ is the MCA a coefficient of the reference category of the $i^{\text {th }}$ factor. The choice between the two methods will therefore hinge upon which aspect of effects, strength or pattern, is more important for meeting the goals of one's research.

\section{EXAMPLE}

An example of both MCA and DVR applied to the same data will illustrate the previous discussion. Data for this example come from the National Opinion Research Center's 1976 General Social Survey. The criterion variable is the NORC racial integration scale which is composed of five items asked only of white respondents. It varies from zero to five with higher scores indicating greater overall willingness to associate with black people. For the sake of brevity, this variable is referred to as "Tolerance" in the following tables and discussion. Three predictors are used: two factors and one covariate. These are Region (coded North, South, and West), Type of Place (coded NonSMSA and SMSA), and respondent's years of education. A complex analysis of covariance allowing interactions showed that no significant interactions were present.

The analysis problem that MCA addresses is: what are the effects of Region and Type of Place upon Tolerance controlling for the linear effect of Education? The results are fairly 
straightforward (Table 1). Region, Type of Place, and Education explain twenty-three percent of the variance in Tolerance, alpha level zero to the third decimal place. Individually, all three predictors are significant to the same alpha level as that of the model as a whole, zero to the third decimal place. Education is the strongest predictor because its beta is larger than the others (.35). Since Education is a covariate, the sign taken by its beta is meaningful, indicating that Tolerance increases with years of education. Of the factors, Region has a stronger overall effect (beta $=.22$ ) upon Tolerance than Type of Place (.12).

Northerners are slightly more tolerant of blacks than average $(\mathrm{a}=.19)$, Westerners even more so (.30). Since these two groups are more tolerant than average, Southerners are less tolerant than average by definition (-.54). As regards Type of Place, SMSA residents are more tolerant than average (.13), implying that NonSMSA residents are less tolerant than average (-.27) controlling for the other predictors in the model. The fact that there will always be at least one positive and one negative value of $a$ for each factor points out the artifactual nature of these coefficients which results from using the grand mean (in this case 3.17) as the reference point.

In contrast to this, DVR addresses the issue of whether or not any of the differences in predicted value between categories of factors are significant. In order to apply DVR to this analysis, a reference category for each factor must be chosen. In this case, North for Region and NonSMSA for Type of Place shall be used. Tolerance is regressed upon four variables: three dummy variables, South, West, and SMSA, and one metric variable, years of education. The results appear in Table 2.

The first thing to note in Table 2 is that the values estimated for the total model using DVR are identical to those estimated in the MCA analysis, underlining the essential equivalence of the two estimation strategies. Years of education produces an F-ratio and beta identical to those of MCA, underlining the fact that covariates are treated identically by both methods.

As regards the betas for the factors in this analysis, the dummy variable SMSA should have a beta whose absolute value is the same as that obtained for Type of Place in the MCA analysis
TABLE 1

MCA analysis of Region, Type of Place, and Education predicting Tolerance

Total Model: Multiple R; 480 Multiple $\mathrm{R}^{2} ; .230$

F-ratio; 91.49 Degrees of freedom; 4/1223 Alpha Level; .000 Grand mean of TOLERANCE: 3.17

\begin{tabular}{|c|c|c|c|c|c|}
\hline Predictor: & Beta: & F: & $\begin{array}{l}\text { Degrees } \\
\text { of freedom: }\end{array}$ & $\begin{array}{l}\text { Alpha } \\
\text { Level: }\end{array}$ & $\begin{array}{c}\text { MCA } \\
\text { coefficient }(a):\end{array}$ \\
\hline REGION & .22 & 46.11 & $2 / 1223$ & .000 & \\
\hline North & & & & & .19 \\
\hline South & & & & & -.54 \\
\hline West & & & & & .30 \\
\hline TYPE OF PLACE & .12 & 46.07 & $1 / 1223$ & .000 & \\
\hline nonSMSA & & & & & -.27 \\
\hline SMSA & & & & & .13 \\
\hline EDUCATION & .35 & 186.39 & $1 / 1223$ & .000 & \\
\hline
\end{tabular}

TABLE 2

DVR analysis of Region, Type of Place, and Education predicting Tolerance

$\begin{aligned} \text { Total Model: } & \text { Multiple } \mathrm{R} ; .480 \quad \text { Multiple } \mathrm{R}^{2} ; .230 \\ & \text { F-ratio; } 91.49 \text { Degrees of freedom; } 4 / 1223 \text { Alpha level: } .000\end{aligned}$
Reference Categories: Region;-NORTH Type of Place; NONSMSA

\begin{tabular}{lcrrrl} 
Predictor: & Beta: & b: & \multicolumn{1}{c}{ F: } & $\begin{array}{c}\text { Degrees } \\
\text { of freedom }\end{array}$ & $\begin{array}{l}\text { Alpha } \\
\text { Level: }\end{array}$ \\
SOUTH & -.21 & -.73 & 62.63 & $1 / 1223$ & .000 \\
WEST & .03 & .10 & .93 & $1 / 1223$ & .335 \\
SMSA & .12 & .39 & 20.58 & $1 / 1223$ & .000 \\
EDUCATION & .35 & .17 & 186.39 & $1 / 1223$ & .000
\end{tabular}

Intercept

TABLE 3

Conversion of MCA a coefficients into DVR b coefficients for Region, Type of Place, and Education predicting Tolerance

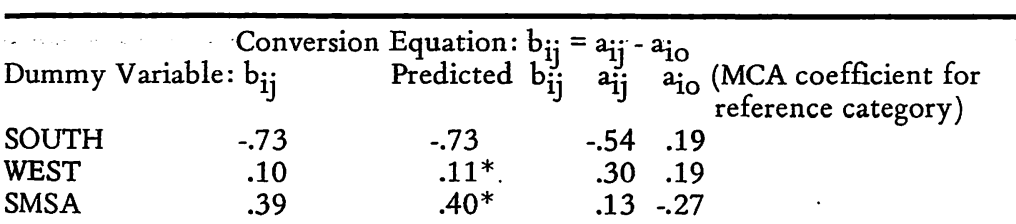

*due to rounding 
because the Type of Place factor is a dichotomy. Indeed, this is the case (beta $=.12$ ). If the dummy variable NonSMSA had been used rather than SMSA, the beta estimated for NonSMSA would have been -.12 since the reference category would then have been SMSA rather than NonSMSA. In the case of a dichotomous factor, the choice of a reference category affects only the sign of the beta and $b$ coefficients. However, the reader will note that there is no such simple relationship between the betas of the dummy variables South and West (-.21 and .03 respectively) and the MCA beta for Region (.22). This is because Region is a polytomy rather than a dichotomy. However, these betas do contribute to the researcher's understanding of what is important in the model. The difference between Southerners and Northerners in tolerance is the most important effect (-.12) after Education, followed by the difference between SMSA and NonSMSA residents. Westerners are slightly more tolerant than Northerners, but this effect is very small (.03) and is not significant (alpha level $=.335$ ). If this regression were to be run again, one might as well delete the dummy variable West, thus expanding the reference category to Westerners and Northerners, i.e., Nonsoutherners.

The actual differences between categories in predicted tolerance appear as $b$ coefficients. Southerners score .73 less on Tolerance, and Westerners score .10 more on Tolerance, than Northerners controlling for Type of Place and Education. SMSA residents score .39 higher on Tolerance than nonSMSA residents controlling for the other variables. Since this is an additive model, one would expect Western SMSA residents, for example, to score $.49(.10+.39)$ higher on Tolerance than Northern nonSMSA residents controlling for Education.

It was stated earlier that when dummy variables are scored zero-one, they could be converted into MCA $a$ coefficients and vice versa. Table 3 shows that this is the case within rounding error.

The author hopes that this discussion and example have helped to clarify the relationship between Multiple Classification Analysis and Dummy Variable Regression.

University of Chicago

Christian Wells Jacobsen
Relationship Between DVR and MCA

\section{REFERENCES}

Andrews, Frank et al.

1973 Multiple Classification Analysis: A Report on a Computer Program for Multiple Regression Using Categorical Predicators, Second Edition. Ann Arbor: Survey Research Center, University of Michigan.

Draper, Norman and Harry Smith

1966 Applied Regression Analysis. New York: John Wiley and Sons, Inc.

Kim, Jae-On and Frank Kohout

1975a "Special Topics in General Linear Models." Pp. 368-97 in Norman Nie et al. (eds.), SPSS, Second Edition. New York: McGraw-Hill Book Co.

1975b "Analyis of Variance and Covariance: Subprograms A NOVA and Oneway." Pp. 398-433 in Norman Nie et al. (eds.), SPSS, Second Edition. New York: McGraw-Hill Book Co. 